\title{
DÉCEMBRE 1973
}

\section{5• ANNÉE - N• 660}
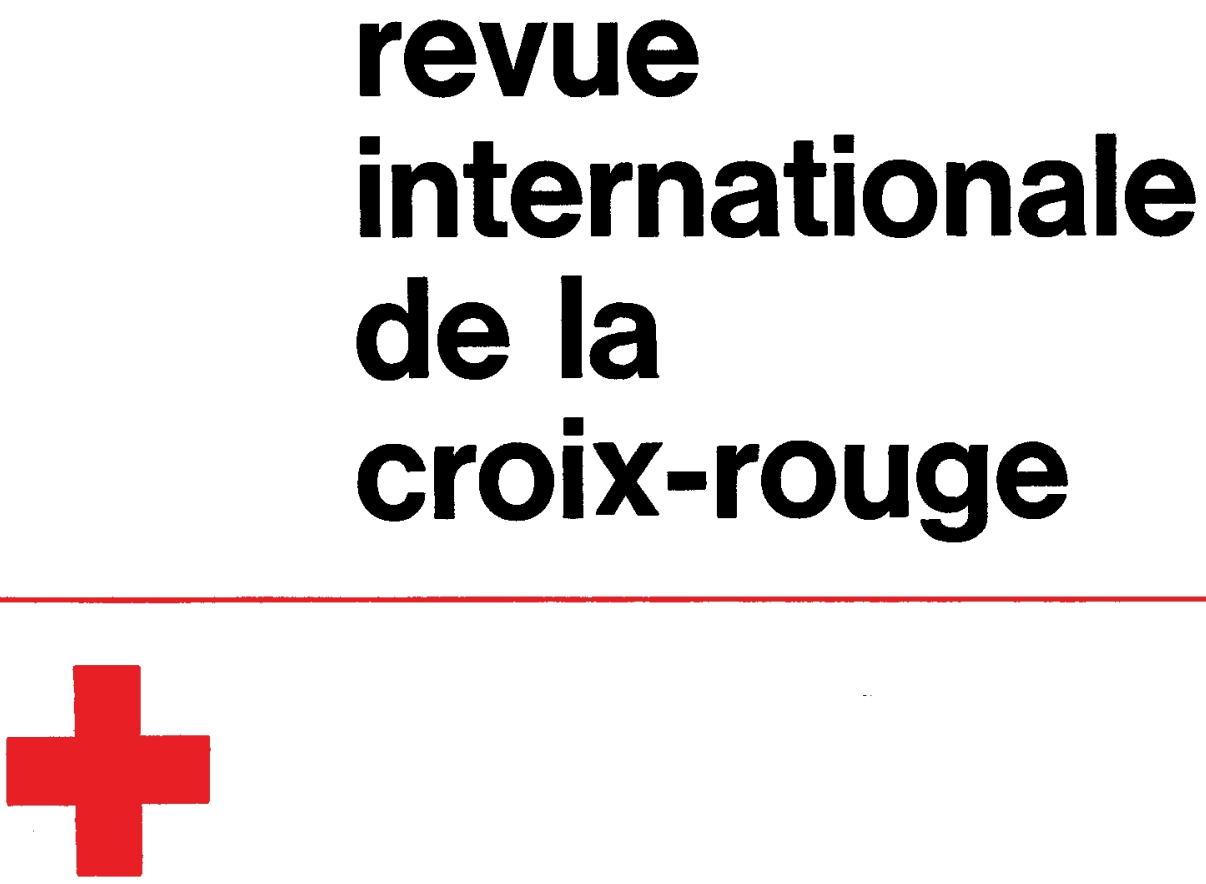

INTER ARMA CARITAS

GENEVE

COMITÉ INTERNATIONAL DE LA CROIX-ROUGE FONDÉ EN 1863 


\title{
COMITÉ INTERNATIONAL DE LA CROIX-ROUGE
}

MM. ERIC MARTIN, docteur en médecine, professeur honoraire de l'Université, Genève, président (membre depuis 1973)

JEAN PICTET, docteur en droit, président de la Commission juridique, vice-président (1967)

HARALD HUBER, docteur en droit, juge fédéral, vice-président (1969)

HANS BACHMANN, docteur en droit, directeur des finances de la ville, Winterthour (1958)

DIETRICH SCHINDLER, docteur en droit, professeur à l'Université, Zurich (1961)

MHe MARJORIE DUVILLARD, infirmière, ancienne directrice del'Ecole d'infirmières du Bon Secours, Genève (1961)

MM. MAX PETITPIERRE, docteur en droit, ancien conseiller fédéral (1961)

ADOLPHE GRAEDEL, ancien député au Conseil national, ancien secrétaire général de la Fédération internationale des ouvriers sur métaux (1965)

$M^{\text {me }}$ DENISE BINDSCHEDLER-ROBERT, docteur en droit, professeur à l'Institut universitaire de hautes études internationales, Genève (1967)

MM. MARCEL A. NAVILLE, licencié ès lettres, président du CICR de 1969 à 1973 (1967)

JACQUES F. DE ROUGEMONT, docteur en médecine (1967)

ROGER GALLOPIN, docteur en droit, ancien directeur général du CICR (1967)

WALDEMAR JUCKER, docteur en droit, secrétaire de 1'Union syndicale suisse (1967)

VICTOR H. UMBRICHT, docteur en droit, administrateur (1970)

PIERRE MICHELI, licencié en droit, ancien ambassadeur (1971)

PIERRE BOISSIER, licencié en droit, directeur de 1'Institut Henry-Dunant, Genève (1973)

GILBERT ETIENNE, docteur en droit, professeur à l'Institut universitaire de hautes études internationales, Genève (1973)

ULRICH MIDDENDORP, docteur en médecine, chef de la clinique chirurgicale de l'Hôpital cantonal, Winterthour (1973)

M" MARION ROTHENBACH, diplômée M.S.W, de l'Université de Mìchigan, maître-assistant à l'Ecole des sciences sociales et politiques de l'Université, Lausanne (1973)

Membres honoraires: M. JACQUES CHENEVIĖRE, vice-président d'honneur; $\mathrm{M}^{\text {Ile }}$ LUCIE ODIER, vice-présidente d'honneur; MM. GUILLAUME BORDIER, CARL J. BURCKHARDT, PAUL CARRY, M ${ }^{\text {me }}$ MARGUERITE GAUTIER-VAN BERCHEM, MM. SAMUEL A. GONARD, EDOUARD DE HALLER, RODOLFO OLGIATI, PAUL RUEGGER, FRÉDERIC SIORDET, ALFREDO VANNOTTI, ADOLF VISCHER.

\section{CONSEIL EXÉCUTIF}

\author{
M. ROGER GALLOPIN, président \\ M. VICTOR H. UMBRICHT, vice-président \\ $M^{m e}$ DENISE BINDSCHEDLER-ROBERT \\ M. GILBERT ETIENNE \\ D ULRICH MIDDENDORP \\ M. GOTTFRIED DE SMIT \\ M. PIERRE MICHELI, membre suppleant
}




\title{
SOCIETE FIDUCIAIRE ROMANDE OFOR S.A.
}

\author{
Place Saint-Gervais 1, Genève - Tél. 317050
}

Expertises, revisions et organisations comptables

\ Domiciliation et administration de sociétés

\ Assainissements et liquidations

- Interventions et conseils en matière fiscale

\section{JOURIAL DE GEIÈV}

deux imprimeries deux adresses

rue François-Dussaud 20

1211 Genève 8 - Téléphone 431940

rue Général-Dufour 5-7

1211 Genève 11 - Téléphone 250350

TYPO - OFFSET - ROTATIVE 


\section{SOMMAIRE}

COMITE INTERNATIONAL DE LA CROIX-ROUGE

DANS LE MONDE DE LA CROIX-ROUGE

\section{FAITS ET DOCUMENTS}

\section{REVUE INTERNATIONALE DE LA CROIX-ROUGE}

DECEMBRE $1973-\mathrm{N}^{\circ} 660$

XXIIre Confërence internationale de la Croix-Rouge

— Discours prononcés à la Séance d'ouverture . 713

L'action du Comité international au Moyen-Orient 727

Activités extérieures:

Chili - Mission en Afrique occidentale - Rhodé-

sie - Mozambique - Sous-continent asiatique 736

A Genève

Un nouveau film du CICR . . . . . . . . . . . . 739

Publications du CICR en langue arabe . . . . . 739

Les réunions de la Croix-Rouge internationale à Téhéran ............. 741

Journée mondiale de la Croix-Rouge - «Sang donné: vie sauvée» . . . . . . . . . . . . 744

Assistance internationale de la Croix-Rouge en Indochine . . . . . . . . . . . . 745

Promouvoir l'image de la Croix-Rouge dans le monde . . . . . . . . . . . 746

Congrès mondial des forces de paix . . . . . 749

Souveraineté nationale et droits de l'homme . . 750

Les infirmières et les Conventions de Genève . 752

Le contrôle des stupéfiants . . . . . . . . 755

Table des matières $1973 \ldots$. . . . . . . . 758 
INTERNATIONAL REVIEW

OF THE RED CROSS

\section{SUPPLEMENTS \\ DE LA REVUE}

EN LANGUE ESPAGNOLE

EN LANGUE ALLEMANDE

REVUE INTERNATIONALE DE LA CROIX-ROUGE
Une édition en langue anglaise paraît chaque mois. Elle est en principe identique à l'édition française, et peut être obtenue aux mêmes conditions.

XXII Conferencia Internacional de la Cruz Roja Una publicación del Comité Internacional de la Cruz Roja — Día Mundial de la Cruz Roja: « Da tu sangre... Salva una vida » - Índice - Vol. XXV (1973).

XXII. Internationale Rotkreuzkonferenz - Ein neuer Film des IKRK - Kolloquium über den seelsorgerischen und den geistigen Beistand in bewaffneten Konflikten und innerstaatlichen Wirren - Ein Kindergesundheitszentrum - Die Wahl fiel auf das Rote Kreuz...! - Inhaltsverzeichnis - Band XXIV (1973).

Elle est publiée chaque mois par le Comité international de la Croix-Rouge.

7, avenue de la Paix, 1211 Genève 1 (Suisse) - Compte de chèques postaux $12-1767$.

Abonnement un an: Fr. 30,-; le numéro: Fr. 3,-.

RÉDACTION : J.-G. LOSSIER

Seuls les textes signés par le Comité international de la Croix-Rouge engagent la responsabilité de celui-ci. 